\section{Embolic Septic Emboli with MRSA: A different source}

\author{
Christian Castillo Latorre*, Mariela Rivera Agosto, Ilean Lamboy \\ Hernandez, Mariana Mercader Perez, Dominic Sanchez Paredes, \\ Marisol Santiago Irizarry, Jose Gutierrez Nuñez Onix, Cantres \\ Fonseca, Alfonso Torres Palacios and William Rodriguez Cintron
}

Pulmonary Medicine and Critical Care Fellowship, VA Caribbean Healthcare System San Juan P.R, USA

\section{More Information}

*Address for Correspondence: Christian Castillo Latorre, Pulmonary Medicine and Critical Care Fellowship, VA Caribbean Healthcare System San Juan P.R, USA, Email: ccl0332@gmail.com

Submitted: 04 December 2019

Approved: 11 December 2019

Published: 12 December 2019

How to cite this article: Latorre CC, Agosto MR, Hernandez IL, Perez MM, Paredes DS, et al. Embolic Septic Emboli with MRSA: A different source. J Clin Intensive Care Med. 2019; 4 : 044-047.

DOI: dx.doi.org/10.29328/journal.jcicm.1001026

ORCiD: orcid.org/0000-0002-0732-6797

Copyright: Latorre CC, et al. This is an open access article distributed under the Creative Commons Attribution License, which permits unrestricted use, distribution, and reproduction in any medium, provided the original work is properly cited.

W) Check for updates

\section{Introduction}

Septic pulmonary embolism (SPE) is an uncommon disorder in which a microorganism-containing thrombus causesanunregulatedinflammatoryreaction, withsubsequent parenchymal destruction. Septic phlebitis consists of purulent material mixed with thrombotic material that is able to travel to organs causing direct damage [1]. Embolization of this material can result in obstruction of pulmonary vessels with subsequent blocked blood flow as well, in which pulmonary ischemia could develop [2]. Pulmonary septic emboli are one of the most common manifestations of hematogenous spread of pathogenic organism such as bacteria or fungi [3]. The source of pathogen could be divided into internal or external, in which documentation of risk factors, previous procedures or lifestyle habits could help in adequate assessment of illness [4]. Internal sources can be seen from an abdominal infection causing subsequent translocation from the gastrointestinal tract or adjacent structures via the venous drainage system. Once hematogenous seeding reaches the heart and endocardium, pulmonary manifestations will be seen in most cases [5]. It's important to note that pulmonary manifestations are not always a sequela of right sided endocarditis. Lower extremity thrombophlebitis is a recognize source of embolization. Assessment of pulmonary manifestations of septic emboli almost always starts ruling out right sided endocarditis with echocardiography [5]. There is no better technique for non-invasive assessment of heart valve vegetations than echocardiography [6]. The detection rate for vegetations by transthoracic echocardiography (TTE) averages around $50 \%$. There are several factors that influence the detection which include vegetation location, size, history 
of prosthetic valves, echogenicity of the vegetation and an experienced examiner [6]. Septic pulmonary emboli are usually associated with right-sided endocarditis or other proximal/distal venous infectious process, such as primary deep tissue infections [6]. One of the most common risk factors for septic pulmonary emboli is intravenous drug usage (IVDA), however patients without IVDA history could develop this sequela as well. The pathogenesis related to pulmonary manifestations relates to venous return to the right side of the heart and subsequent seeding of microorganism into the lung vasculature. One of the most common organism known to cause this phenomenon is MRSA. However, there are multiple other etiologic organisms depending on the primary source of infection or risk factors [7].

Septicemia due to iliac vein thrombophlebitis is a direct source of bacterial seeding from the abdomen into the right side of the heart. Venous embolic phenomena from iliac veins has an insidious onset of events which could cause severe acute respiratory distress syndrome [8]. The pathogenesis of acute respiratory distress syndrome in the setting of septic emboli relates to systemic inflammatory reaction with localized alveolar damage and subsequent impairment of ventilation [8]. This acute inflammatory response to a bacterial organism makes prompt initiation of effective antibiotic therapy a critical role in management. In some cases, delayed in recognition could lead to an overwhelming response [7]. Cytokine production related to lung insult could add to the worsening scenario that mechanical ventilation induced volutrauma may cause. Most patients with septic pulmonary emboli are diagnosed on the basis of computer tomography findings such as peripheral nodules with or without cavitary lesions, which may cause abscess formation [9]. The aims of treatment are to prevent the septic thrombus from further embolization. Thrombus removal which is the primary source of sepsis should be considered. Treatment options such as mechanical thrombectomy, directed thrombolysis, endovascular approach or surgical thrombectomy have been described [10].

\section{Case Report}

A 61-year-old man with past medical history of coronary artery disease, dyslipidemia and chronic back pain, no history of substance abuse came to the ED. He had previous ED visits due to back pain. In all previous ED visits multiple, intramuscular systemic steroids and pain control medications have been administered with adequate relief of symptoms. However, the patient developed bilateral lower extremity weakness episodes, which was the main concern at time of evaluation in our institution. He was afebrile with an adequate rate of $100 / \mathrm{min}$ and respiratory rate of $20 /$ min. Physical examination revealed 5/5 strength in upper extremities and $4 / 5$ strength in bilateral lower extremities. He had a white blood cell count of $11,500 \times 10^{3} / \mathrm{mcL}$ with an absolute neutrophil count of $78 \%$, bands $5 \%$, and hemoglobin of 14.6. Chest (CT) scan showed innumerable nodular and patchy focal densities throughout the lungs, many of which demonstrated internal cavitation or internal groundglass appearance (Figures 1,2). Lumbar computer tomography (CT) showed an abnormal prevertebral soft tissue projecting from L5 to S1, findings could be secondary to underlying infectious spondylitis changes of the L5 - S1 disc space level (Figure 3). Lumbar spine degenerative changes; and a L3 vertebral body hemangioma. Cervical CT showed extensive bilateral pulmonary parenchymal abnormalities multifocal nodularities and opacities some of them peripheral and with internal lucencies.

Blood culture came back to be positive for gram-positive cocci which was later confirmed to be MRSA infection. With acutely worsening of respiratory pattern and subsequent hypoxemia the patient was intubated, meeting criteria for severe acute respiratory distress syndrome. Further lumbar

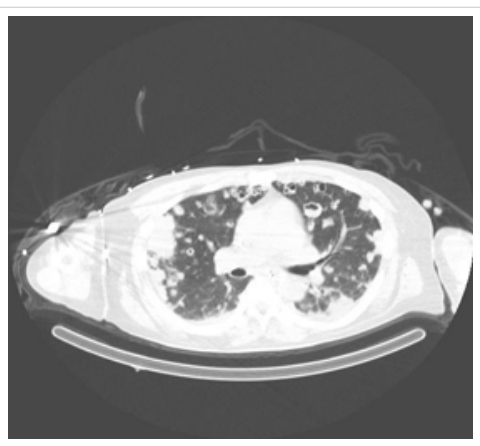

Figure 1: Chest computer tomography without contrast showing bilateral cavitary pulmonary emboli.

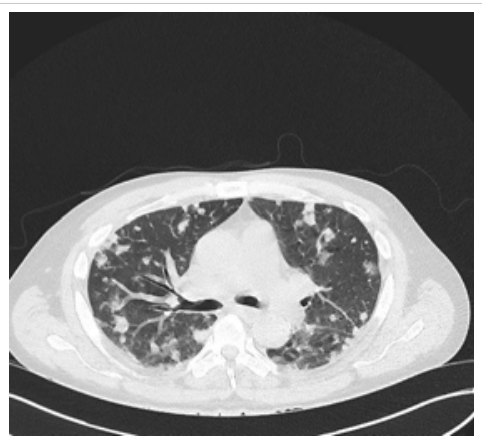

Figure 2: Chest computer tomography without contrast showing bilateral cavitary pulmonary emboli.

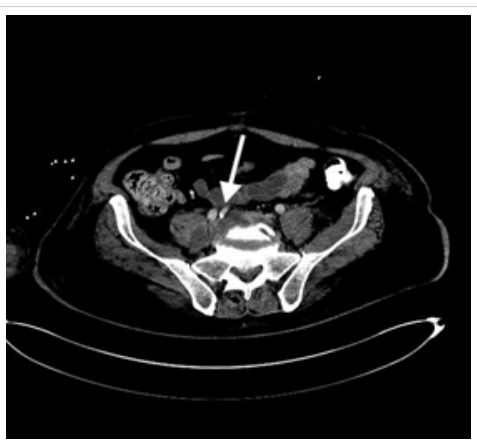

Figure 3: Abdominopelvic computer tomography without contrast showing psoas abscess with associated diskitis and iliac vein thrombophlebitis. 
studies demonstrated iliac vein thrombosis with adjacent psoas abscess. Psoas abscess was unable to be drained due to its location. Adjacent iliac vein thrombophlebitis was subsequently treated with full anticoagulation. Due to critical state thrombolysis and other approaches were not initiated. These imaging findings and her staphylococcal bacteremia raised suspicion for infective endocarditis. However, no embolic features were present on physical examination of the extremities or eye fundus. In addition to this a transesophageal echocardiogram demonstrated no valve abnormality, thus effectively ruling out infective endocarditis. The patient was started on IV vancomycin initially, double coverage for MRSA was started in view of extensive disease. Negative blood cultures were reached after 1 month of therapy. After 3 months of aggressive early mobility, multiple antibiotics, and lung protective ventilation the patient was discharged from our institution and currently is undergoing successful physical rehabilitation.

\section{Discussion}

Septic Deep Vein Thrombosis has been reported as a source of septic pulmonary embolism. It is well recognized that intravenous drug abusers (IVDA) commonly present with skin or subcutaneous abscesses and suppurative thrombophlebitis of veins, our patient denied such IVDA [11]. However, intramuscular administration of medications could have lead to distant thrombophlebitis and subsequent abscess formation. Psoas abscess formation due to bacterial seeding with adjacent iliac vein thrombophlebitis was the detonating event of this pulmonary sequela that caused ARDS.

In our case, SA is the predominant infectious agent. However, Gram-negative bacilli or fungi, are more prevalent in patients with nosocomial, catheter-related suppurative thrombophlebitis [12]. The choice and administration of initial empiric antibiotic therapy should include a broadspectrum coverage [13]. It's imperative to maintain adequate blood antibiotic levels for adequate clearance, close monitoring of toxicity and through levels as well [13]. Concentration dependent pharmacokinetics always plays a role in this type of complicated patients in which persistent bacteremia could be seen due to inability of infectious focus source control [14]. The duration and choice of antibiotics was based on the clinical evolution, serological infection markers and drug side effects. According to treatment guidelines, it is recommended that bacteremia could be treated for 14 days. However, in our patient decision was made to be treated for 4 to 6 weeks in view of thrombophlebitis with associated psoas abscess. Decisions regarding thrombus therapy should be multidisciplinary in view of the risk to benefit of possible bleeding with anticoagulation. In our case we opted for full anticoagulation, and no surgical procedures or further noninvasive procedures.
The physiology and complications of bed rest are well understood, and for our ICU team is one of the primary preventive goals. Intensive care unit-acquired weakness and functional dependency are recognized as unfortunate consequences of prolonged ICU admissions and many of them requiring mechanical ventilation [15]. In our case early mobility rehabilitation protocol was a major priority for which the patient received active reassurance by the mobility team as well by physicians on charge of the case. All of which facilitated early patient move, early mobilization made possible preservation of muscle strength and regaining of quality of life respectively. Aggressive daily orientation technique facilitated for less sedative agent use, better spontaneous breathing and spontaneous awakening trials [16]. There was no occurrence during the month the patient stayed at the ICU of hyperactive or hypoactive delirium.

\section{Conclusion}

In conclusion, septic deep vein thrombosis is a rare recurrence with severe sequelae if not recognized and hence treated early and aggressively. The use of intravenous or any other route of medications administration, is an important clue in the differential diagnosis. This information could lead for the primary source of infection, which in this case was of vessel (thrombophlebitis) etiology. Disease led to serious complications associated with ARDS, which in this case were successfully managed following guidelines. The ICU is the place where multidisciplinary approach warrants the best patient's outcomes. Early mobility physical therapy is an example which added to a functional, successful outcome of a complex case.

\section{References}

1. Losno RA, Vidal-Sicart S, Grau JM. Multiple pyomyositis secondary to septic thrombophlebitis. Med Clin (Barc). 2019; 152: 515-516. PubMed: https://www.ncbi.nlm.nih.gov/pubmed/30340839

2. Stawicki SP, Firstenberg MS, Lyaker MR, Russell SB, Evans DC, et al. Septic embolism in the intensive care unit. Int J Crit IIIn Inj Sci. 2013; 3: 58-63.

PubMed: https://www.ncbi.nlm.nih.gov/pubmed/23724387

3. Ye R, Zhao L, Wang C, Wu X, Yan H. Clinical characteristics of septic pulmonary embolism in adults: A systematic review. Respir Med. 2013; 108: 1-8.

PubMed: https://www.ncbi.nlm.nih.gov/pubmed/24183289

4. Cook RJ, Ashton RW, Aughenbaugh GL, Ryu JH. Septic pulmonary embolism: presenting features and clinical course of 14 patients. Chest. 2005; 128: 162-166.

PubMed: https://www.ncbi.nlm.nih.gov/pubmed/16002930

5. Baddour LM, Wilson WR, Bayer AS, Fowler VG Jr, Tleyjeh IM, et al. Infective endocarditis in adults: diagnosis, antimicrobial therapy, and management of complications: a scientific statement for healthcare professionals from the American Heart Association. Circulation. 2015; 132: 1435-1486

PubMed: https://www.ncbi.nlm.nih.gov/pubmed/26373316

6. Greaves K, Mou D, Patel A, Celermajer DS. Clinical criteria and the appropriate use of transthoracic echocardiography for the exclusion of infective endocarditis. Heart. 2003; 89: 273-275. 
7. Green RS, Travers AH, Cain E, Campbell SG, Jensen JL, et al. Paramedic Recognition of Sepsis in the Prehospital Setting: A Prospective Observational Study. Emerg Med Int. 2016; 2016; 2016.

8. Prnjavorac B, Sejdinovic R, Jusufovic E, Mehic J, Bedak O, et al. Septic pulmonary embolisms as a cause of acute respiratory distress syndrome. Euro Respiratory J. 2016; 48.

9. Kuhlman JE, Fishman EK, Teigen C. Pulmonary septic emboli: Diagnosis with CT. Radiology. 1990; 174: 211-213.

PubMed: https://www.ncbi.nlm.nih.gov/pubmed/2294550

10. Kniemeyer HW, Grabitz K, Buhl R, Wüst HJ, Sandmann W. Surgical treatment of septic deep venous thrombosis. Surgery. 1995; 118: 49-53. PubMed: https://www.ncbi.nlm.nih.gov/pubmed/7604379

11. Zuo LE, Guo S. Septic pulmonary embolism in intravenous drug users. Chin J Tuberc Respir Dis 2007; 30: 569-572.

PubMed: https://www.ncbi.nlm.nih.gov/pubmed/17988547

12. Brenes JA, Goswami U, Williams DN. The association of septic thrombophlebitis with septic pulmonary embolism in adults. Open Respir Med J. 2012; 6: 14-19.

PubMed: https://www.ncbi.nlm.nih.gov/pubmed/22611460

13. Mara Lambert. IDSA Guidelines on the Treatment of MRSA Infections in Adults and Children. Am Fam Physician. 2011; 84: 455-463.

14. Shah S, Barton G, Fischer A. Pharmacokinetic considerations and dosing strategies of antibiotics in the critically ill patient. J Intensive Care Soc. 2015; 16: 147-153.

PubMed: https://www.ncbi.nlm.nih.gov/pubmed/28979397

15. Hodgson CL, Capell E, Tipping CJ. Early Mobilization of Patients in Intensive Care: Organization, Communication and Safety Factors that Influence Translation into Clinical Practice. Crit Care. 2018; 22: 77. PubMed: https://www.ncbi.nlm.nih.gov/pubmed/29558969

16. Brummel NE, Girard TD. Preventing delirium in the intensive care unit. Crit Care Clin. 2013; 29: 51-65.

PubMed: https://www.ncbi.nlm.nih.gov/pubmed/23182527 